\title{
A NOTE ON LATTICES WITH MANY SUBLATTICES
}

\author{
GÁBOR CZÉDLI AND ESZTER K. HORVÁTH
}

Received 20 January, 2019

\begin{abstract}
For every natural number $n \geq 5$, we prove that the number of subuniverses of an $n$ element lattice is $2^{n}, 13 \cdot 2^{n-4}, 23 \cdot 2^{n-5}$, or less than $23 \cdot 2^{n-5}$. Also, we describe the $n$-element lattices with exactly $2^{n}, 13 \cdot 2^{n-4}$, or $23 \cdot 2^{n-5}$ subuniverses.
\end{abstract}

2010 Mathematics Subject Classification: 06B99

Keywords: finite lattice, sublattice, number of sublattices, subuniverse

\section{INTRODUCTION AND OUR RESULT}

For a lattice $L, \operatorname{Sub}(L)$ will denote its sublattice lattice. In spite of this standard terminology, $\operatorname{Sub}(L)$ consists of all subuniverses of $L$. That is, a subset $X$ of $L$ is in $\operatorname{Sub}(L)$ iff $X$ is closed with respect to join and meet. In particular, $\varnothing \in \operatorname{Sub}(L)$. All lattices occurring in this paper will be assumed to be finite even if this is not always emphasized. For a natural number $n \in \mathbb{N}^{+}:=\{1,2,3, \ldots\}$, let

$$
\operatorname{NS}(n):=\{|\operatorname{Sub}(L)|: L \text { is a lattice of size }|L|=n\} .
$$

That is, $k \in \mathrm{NS}(n)$ if and only if some $n$-element lattice has exactly $k$ subuniverses. Although the acronym NS comes from Number of Sublattices, $L$ has only $|\operatorname{Sub}(L)|-$ 1 sublattices. If $K$ and $L$ are finite lattices, then their glued sum $K+{ }_{g l u} L$ is the ordinal sum of the posets $K \backslash 1_{K}$, the singleton lattice, and $L \backslash\left\{0_{L}\right\}$, in this order. In other words, we put $L$ atop $K$ and identify the elements $1_{K}$ and $0_{L}$; see Figure 1 . For example, if each of $K$ and $L$ is the two-element chain, then $K+{ }_{g l u} L$ is the threeelement chain. Note that $+_{g l u}$ is an associative but not a commutative operation.

The following fact is trivial:

The largest number in $\mathrm{NS}(n)$ is $2^{n}=32 \cdot 2^{n-5}$.

Furthermore, an $n$-element lattice $L$ has exactly $2^{n}$ subuniverses if an only if $L$ is a chain.

This research was supported by the Hungarian Research Grants KH 126581. 

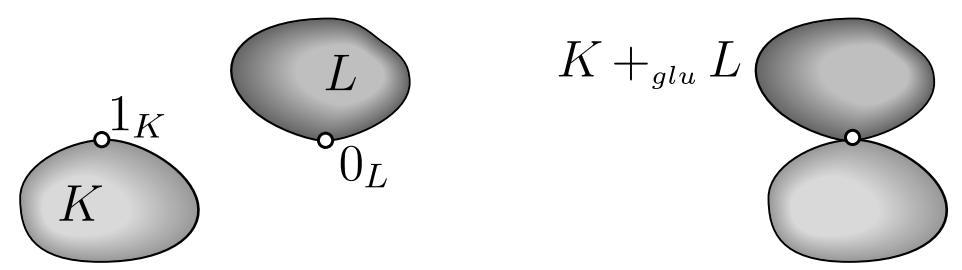

FIGURE 1. The glued sum $K+{ }_{g l u} L$ of $K$ and $L$

The four-element boolean lattice $B_{4}$ and the pentagon (lattice) $N_{5}$ are given in Figure 2, together with other important lattices. For $k \in \mathbb{N}^{+}$, the $k$-element chain will be denoted by $C^{(k)}$. Our goal is to prove the following result:

Theorem 1. If $5 \leq n \in \mathbb{N}^{+}$, then the following three assertions hold.

(1) The second largest number in $\mathrm{NS}(n)$ is $26 \cdot 2^{n-5}$. Furthermore, an n-element lattice $L$ has exactly $26 \cdot 2^{n-5}$ subuniverses if and only if $L \cong C_{1}+{ }_{g l u} B_{4}$ $+{ }_{g l u} C_{2}$, where $C_{1}$ and $C_{2}$ are chains.

(2) The third largest number in $\mathrm{NS}(n)$ is $23 \cdot 2^{n-5}$. Furthermore, an n-element lattice $L$ has exactly $23 \cdot 2^{n-5}$ subuniverses if and only if $L \cong C_{0}+{ }_{g l u} N_{5}$ ${ }_{\text {glu }} C_{1}$, where $C_{0}$ and $C_{1}$ are chains.

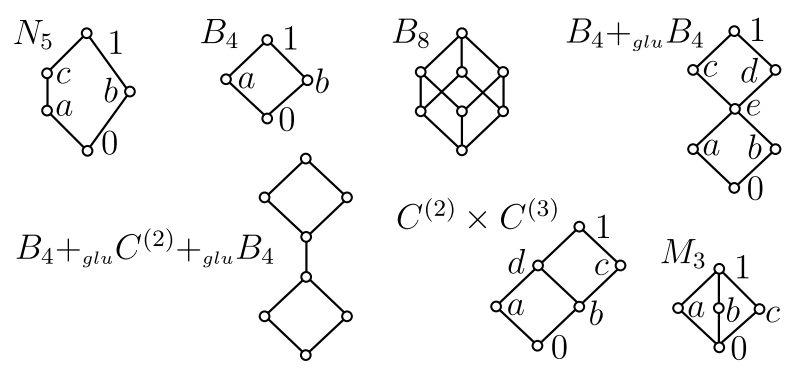

FIGURE 2. Lattices for Theorem 1 and Lemma 2

Since $\operatorname{NS}(n)=\left\{2^{n}\right\}$ for $n \in\{1,2,3\}$ and NS(4) $=\{13,16\}$, we have formulated this theorem only for $n \geq 5$. To make the comparison of the numbers occurring in the paper easier, we often give $|\operatorname{Sub}(L)|$ as a multiple of $2^{|L|-5}$. Next, we repeat the first sentence of the Abstract, which is a trivial consequence of Theorem 1.

Corollary 1. For $5 \leq n \in \mathbb{N}^{+}$, the number of subuniverses of an $n$-element lattice is $2^{n}, 13 \cdot 2^{n-4}, 23 \cdot 2^{n-5}$, or less than $23 \cdot 2^{n-5}$.

Remark 1. Let $\operatorname{Con}(L)$ and $\mathrm{NC}(n)$ stand for the lattice of congruences of a lattice $L$ and $\{|\operatorname{Con}(L)|: L$ is a lattice with $|L|=n\}$, respectively. For $n \geq 5$, the five largest numbers in $\mathrm{NC}(n)$ are $16 \cdot 2^{n-5}, 8 \cdot 2^{n-5}, 5 \cdot 2^{n-5}, 4 \cdot 2^{n-5}$, and $3.5 \cdot 2^{n-5}$ by Freese [4], Czédli [2] and, mainly, Kulin and Mureşan [6]. 
Remark 2. Interestingly, the first three of the five numbers mentioned in Remark 1 are witnessed exactly by the same lattices that occur in Theorem 1 and (1.1). However, we will show at the end of Section 2 that

$$
\begin{aligned}
\left|\operatorname{Sub}\left(N_{5}+{ }_{g l u} C^{(3)}\right)\right| & =23 \cdot 2^{7-5}>21.25 \cdot 2^{7-5} \\
& =\left|\operatorname{Sub}\left(B_{4}+{ }_{g l u} B_{4}\right)\right|>19 \cdot 2^{7-5} \\
& =\left|\operatorname{Sub}\left(\left(C^{(2)} \times C^{(3)}\right)+{ }_{g l u} C^{(2)}\right)\right|,
\end{aligned}
$$

which indicates that $\left|\operatorname{Sub}\left(\left(C^{(2)} \times C^{(3)}\right)+{ }_{g l u} C^{(2)}\right)\right|$ is not the fourth largest number in NS(7), although we know from Kulin and Mureşan [6] that $\mid \mathrm{Con}\left(\left(C^{(2)} \times\right.\right.$ $\left.\left.C^{(3)}\right)+{ }_{g l u} C^{(2)}\right) \mid$ is the fourth largest number in $\mathrm{NC}(7)$.

While there are powerful tools to determine the first few large numbers in $\mathrm{NC}(n)$, see the above-mentioned papers and, for additional tools, Czédli [3], the analogous task for NS $(n)$ seems to be more tedious. This together with Remark 2 are our excuses that we do not determine the fourth and fifth largest numbers in NS(n).

The rest of the paper is devoted to the proof of Theorem 1.

\section{TWO PREPARATORY LEMMAS}

Our notation and terminology is standard, see, for example, Grätzer [5]. However, we recall some notation and introduce some auxiliary concepts. For elements $u, v$ in a lattice $L$, the interval $[u, v]:=\{x \in L: u \leq x \leq v\}$ is defined only if $u \leq v$, but the sublattice $[\{u, v\}]$ generated by $\{u, v\}$ always makes sense. In order to avoid confusion, the curly brackets are never omitted from $\left[\left\{a_{1}, \ldots, a_{k}\right\}\right]$ when a generated sublattice is mentioned. For $u \in L$, the principal ideal and the principal filter generated by $u$ are $\downarrow u:=\{x \in L: x \leq u\}$ and $\uparrow u:=\{x \in L: u \leq x\}$, respectively. We can also write $\downarrow_{L} u$ and $\uparrow_{L} v$ to specify the lattice $L$. For $u, v \in L$, we write $u \| v$ if $u$ and $v$ are incomparable, that is, $u \not \leq v$ and $v \not \leq u$. We say that $u$ is join-irreducible if $u$ has at most one lower cover; note that $0=0_{L}$ is join-irreducible by our convention. Meet-irreducibility is defined dually, and an element is doubly irreducible if it is both join-irreducible and meet-irreducible. Next, let us call an element $u \in L$ isolated if $u$ is doubly irreducible and $L=\downarrow u \cup \uparrow u$. That is, if $u$ is doubly irreducible and $x \| u$ holds for no $x \in L$. Finally, an interval $[u, v]$ will be called an isolated edge if it is a prime interval, that is, $u \prec v$, and $L=\downarrow u \cup \uparrow v$.

Lemma 1. If $K$ is a sublattice and $H$ is a subset of a finite lattice $L$, then the following three assertions hold.

(1) With the notation $t:=|\{H \cap S: S \in \operatorname{Sub}(L)\}|$, we have that $|\operatorname{Sub}(L)| \leq$ $t \cdot 2^{|L|-|H|}$.

(2) $|\operatorname{Sub}(L)| \leq|\operatorname{Sub}(K)| \cdot 2^{|L|-|K|}$. 
(3) Assume, in addition, that $K$ has neither an isolated element, nor an isolated edge. Then $|\operatorname{Sub}(L)|=|\operatorname{Sub}(K)| \cdot 2^{|L|-|K|}$ if and only if $L$ is (isomorphic to) $C_{0}+{ }_{\text {glu }} K+{ }_{\text {glu }} C_{1}$ for some chains $C_{0}$ and $C_{1}$.

Proof. With respect to the map $\varphi: \operatorname{Sub}(L) \rightarrow\{H \cap S: S \in \operatorname{Sub}(L)\}$, defined by $X \mapsto H \cap X$, each $Y \in\{H \cap S: S \in \operatorname{Sub}(L)\}$ has at most $2^{|L|-|H|}$ preimages. This yields part (1). Clearly, (1) implies (2). The argument above yields a bit more than stated in (1) and (2); namely, for later reference, note the following.

$$
\begin{aligned}
& \text { If }|\operatorname{Sub}(L)|=|\operatorname{Sub}(K)| \cdot 2^{|L|-|K|} \text {, then for every } \\
& S \in \operatorname{Sub}(K) \text { and every subset } X \text { of } L \backslash K \text {, we have } \\
& \text { that } S \cup X \in \operatorname{Sub}(L) \text {. }
\end{aligned}
$$

Next, we claim that for an element $u \in L$,

$u$ is isolated if and only if for every $X \in \operatorname{Sub}(L)$, we have that $X \cup\{u\} \in \operatorname{Sub}(L)$ and $X \backslash\{u\} \in \operatorname{Sub}(L)$.

Assume that $u$ is isolated and $X \in \operatorname{Sub}(L)$. Since $u$ is doubly irreducible, $X \backslash\{u\} \in$ $\operatorname{Sub}(L)$. Since $u$ is comparable with all elements of $X, X \cup\{u\} \in \operatorname{Sub}(L)$, proving the "only if" part of (2.2). To show the converse, assume that $u$ is not isolated. If $u$ is not doubly irreducible, then $u=a \vee b$ with $a, b<u$ or dually, and $X:=\{a, b, u, a \wedge$ $b\} \in \operatorname{Sub}(L)$ but $X \backslash\{u\} \notin \operatorname{Sub}(L)$. If $u \| v$ for some $v \in L$, then $\{v\} \in \operatorname{Sub}(L)$ but $\{v\} \cup\{u\} \notin \operatorname{Sub}(L)$. This proves the "if" part, and (2.2) has been verified.

Next, to prove part (3), assume that $K$ has neither an isolated element, nor an isolated edge. First, let $L=C_{1}+{ }_{g l u} K+_{g l u} C_{2}$. Since every $u$ in $L \backslash K$ is clearly an isolated element of $L$, it follows from a repeated application of (2.2) that whenever $X \subseteq L \backslash K$ and $S \in \operatorname{Sub}(K)$, then $S \cup X \in \operatorname{Sub}(L)$. Since $L \backslash K$ has $2^{|L|-|K|}$ subsets, $|\operatorname{Sub}(L)| \geq|\operatorname{Sub}(K)| \cdot 2^{|L|-|K|}$, and we obtain the required equality by the converse inequality given in part (2).

Conversely, assume the equality given in (3). Let $x$ be an arbitrary element of $L \backslash K$. Applying (2.1) to $\left\{0_{K}\right\} \in \operatorname{Sub}(K)$ and $\left\{1_{K}\right\} \in \operatorname{Sub}(K)$, we obtain that both $\left\{0_{K}, x\right\}$ and $\left\{1_{K}, x\right\}$ are in $\operatorname{Sub}(L)$, whence neither $x \| 0_{K}$, nor $x \| 1_{K}$. So exactly one of the cases $0_{K}<x<1_{K}, x<0_{K}$, and $1_{K}<x$ holds; we are going to exclude the first one. Suppose for a contradiction that $0_{K}<x<1_{K}$. Then $x$ is comparable to every $y \in K$, because otherwise $S:=\{y\}$ and $\{x\}$ would violate (2.1). By finiteness, we can take $u:=\bigvee(K \cap \downarrow x)$ and $v:=\bigwedge(K \cap \uparrow x)$. Now if $y \in K$, then either $y>x$ and so $y \in \uparrow_{K} v$, or $y<x$ and so $y \in \downarrow_{K} u$, which means that $K=\downarrow_{K} u \cup \uparrow_{K} v$. Hence, $[u, v]_{K}$ is a prime interval of $K$, and so it is an isolated edge of $K$. This is a contradiction, which excludes that $0_{K}<x<1_{K}$. Therefore, with the notation $C_{0}:=$ $\downarrow_{L} 0_{K}$ and $C_{1}:=\uparrow_{L} 1_{K}$, we obtain that $L$ is (isomorphic to) $C_{0}+{ }_{g l u} K+{ }_{g l u} C_{1}$. Consequently, in order to show that $C_{0}$ and $C_{1}$ are chains and to complete the proof, it suffices to show that every $u \in L \backslash K$ is an isolated element of $L$. Suppose the contrary. Then (2.2) yields a subuniverse $Y \in \operatorname{Sub}(L)$ such that

$$
Y \cup\{u\} \notin \operatorname{Sub}(L) \quad \text { or } \quad Y \backslash\{u\} \notin \operatorname{Sub}(L) .
$$


Since $u \notin K$, we have that $Y \cap K=(Y \cup\{u\}) \cap K=(Y \backslash\{u\}) \cap K$; we denote this set by $S$. Then $S \in \operatorname{Sub}(K)$ since $Y \in \operatorname{Sub}(L)$. It follows from (2.1) that

$$
\begin{aligned}
& Y \cup\{u\}=S \cup((Y \cup\{u\}) \backslash K) \in \operatorname{Sub}(L) \text { and } \\
& Y \backslash\{u\}=S \cup((Y \backslash\{u\}) \backslash K) \in \operatorname{Sub}(L),
\end{aligned}
$$

which contradicts (2.3) and completes the proof of Lemma 1

The following lemma is easier and even a computer program could prove it. For the reader's convenience, we give its short proof.

Lemma 2. For the lattices given in Figure 2, the following seven assertions hold.

(1) $\left|\operatorname{Sub}\left(B_{4}\right)\right|=13=26 \cdot 2^{4-5}$.

(2) $\left|\operatorname{Sub}\left(N_{5}\right)\right|=23=23 \cdot 2^{5-5}$.

(3) $\left|\operatorname{Sub}\left(C^{(2)} \times C^{(3)}\right)\right|=38=19 \cdot 2^{6-5}$.

(4) $\left|\operatorname{Sub}\left(B_{4}+{ }_{\text {glu }} B_{4}\right)\right|=85=21.25 \cdot 2^{7-5}$.

(5) $\left|\operatorname{Sub}\left(B_{4}+{ }_{\text {glu }} C^{(2)}+{ }_{\text {glu }} B_{4}\right)\right|=169=21.125 \cdot 2^{8-5}$.

(6) $\left|\operatorname{Sub}\left(M_{3}\right)\right|=20=20 \cdot 2^{5-5}$.

(7) $\left|\operatorname{Sub}\left(B_{8}\right)\right|=74=9.25 \cdot 2^{8-5}$.

Proof. The notation given by Figure 2 will extensively be used.

Among all subsets of $B_{4}$, only $\{a, b\},\{a, b, 0\}$, and $\{a, b, 1\}$ are not subuniverses; this proves (1). Implicitly, Lemma 1(3) will often be used below. Observe that

$$
\begin{aligned}
& \left|\left\{S \in \operatorname{Sub}\left(N_{5}\right):\{a, c\} \cap S=\varnothing\right\}\right|=8, \quad \text { by }(1.1), \\
& \left|\left\{S \in \operatorname{Sub}\left(N_{5}\right):\{a, c\} \cap S \neq \varnothing, b \notin S\right\}\right|=3 \cdot 4=12, \text { and } \\
& \left|\left\{S \in \operatorname{Sub}\left(N_{5}\right):\{a, c\} \cap S \neq \varnothing, b \in S\right\}\right|=3,
\end{aligned}
$$

whereby $\left|\operatorname{Sub}\left(N_{5}\right)\right|=8+12+3=23$ proves (2). Next, $S$ will belong to $\operatorname{Sub}\left(C^{(2)} \times C^{(3)}\right)$ even if this is not indicated. Let us compute:

$$
\begin{aligned}
& |\{S: a \notin S\}|=26, \quad \text { by Lemmas } 1(3) \text { and } 2(1), \\
& |\{S: a, b \in S\}|=3, \text { since } 0, d \in S \text { and } c \in S \Rightarrow 1 \in S, \\
& |\{S: a \in S, b \notin S, c \in S\}|=1, \text { since } 0,1 \in S \text { and } d \notin S, \\
& |\{S: a \in S, b \notin S, c \notin S\}|=8 \text {, by (1.1). }
\end{aligned}
$$

Hence, $26+3+1+8=38$ proves (3). Next, $S$ will automatically belong to $\operatorname{Sub}\left(B_{4}+{ }_{g l u} B_{4}\right)$. We have that $\mid\{S:\{a, b\} \subseteq S \mid\}=7$, because then $\{c, d\} \subseteq S \Rightarrow$ $1 \in S$ and $0, e \in S$. Also, $\mid\{S:\{a, b\} \nsubseteq S \mid\}=13 \cdot 3 \cdot 2=78$, because Lemma 2(1) applies to the upper $B_{4}$, there are 3 possibilities for $a$ and $b$, and two for 0 . Hence, $78+7=85$ proves (4). For $S \in \operatorname{Sub}\left(B_{4}+{ }_{g l u} C^{(2)}+{ }_{g l u} B_{4}\right)$, the intersection of $S$ with the lower $B_{4}$ and that with the upper $B_{4}$ can independently be chosen. Therefore, (5) follows from (1). 
Next, we count the subuniverses $S$ of $M_{3}$. There are 4 with the property $\mid\{a, b, c\} \cap$ $S \mid \geq 2$, because they contain 0 and 1 . There are $3 \cdot 4=12$ with $|\{a, b, c\} \cap S|=1$, and 4 with $|\{a, b, c\} \cap S|=0$. Thus, $\left|\operatorname{Sub}\left(M_{3}\right)\right|=4+12+4=20$, proving (6).

The argument for $B_{8}$ is more tedious. It has 9 at most one-element subuniverses. There are 12 edges. We have 6 two-element subuniverses in which the heights of the two elements differ by two and 1 in which this difference is three. We have 12 three-element covering chains and 6 non-covering ones. The number of four-element (necessarily covering) chains is $3 \cdot 2=6, B_{4}$ is embedded in 6 cover-preserving ways and (thinking of pairs of complementary elements) in 3 additional ways. The fiveelement sublattices are obtained from cover-preserving $B_{4}$-sublattices by adding the unique one of $0_{B_{8}}$ or $1_{B_{8}}$ that is missing; their number is 6 . To each of the $B_{4}$ sublattices at the bottom we can glue a $B_{4}$-sublattice at the top in two ways, whence there are exactly 6 six-element subuniverses. In absence of doubly irreducible elements, there is no seven-element sublattice, and there is 1 eight-element one. The sum of the numbers we have listed is 74, proving (7) and Lemma 2

Now, we are in the position to prove (1.2), mentioned in Remark 2.

Proof of Remark 2. We obtain the statement by combining Lemma 1(3) with parts (2), (3), and (4) of Lemma 2.

\section{THE REST OF THE PROOF}

For brevity, a $k$-element antichain will be called a $k$-antichain. First, we recall two well-known facts from the folklore.

Lemma 3. For every join-semilattice $S$ generated by $\{a, b, c\}$, there is a unique surjective homomorphism $\varphi$ from the free join-semilattice $F_{\mathrm{js}}(\tilde{a}, \tilde{b}, \tilde{c})$, given in Figure 3, onto $S$ such that $\varphi(\tilde{a})=a, \varphi(\tilde{b})=b$, and $\varphi(\tilde{c})=c$.
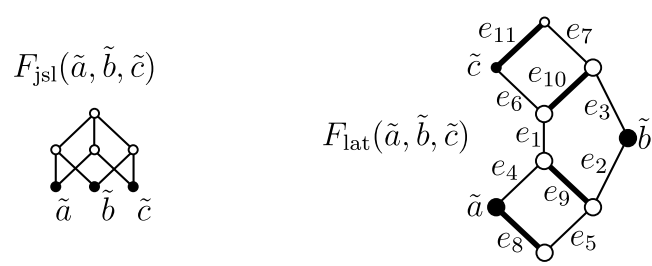

FIGURE 3. $F_{\mathrm{jsl}}(\tilde{a}, \tilde{b}, \tilde{c})$ and $F_{\mathrm{lat}}(\tilde{a}, \tilde{b}, \tilde{c})$

Lemma 4 (Rival and Wille [7, Figure 2]). For every lattice $K$ generated by $\{a, b, c\}$ such that $a<c$, there is a unique surjective homomorphism $\varphi$ from the finitely presented lattice $F_{\text {lat }}(\tilde{a}, \tilde{b}, \tilde{c})$, given in Figure 3 , onto $K$ such that $\varphi(\tilde{a})=a, \varphi(\tilde{b})=b$, and $\varphi(\tilde{c})=c$. 
The subscripts in the notations are explained by the facts that $F_{\mathrm{js}}(\tilde{a}, \tilde{b}, \tilde{c})$ is the free join-semilattice on 3 generators, while $F_{\text {lat }}(\tilde{a}, \tilde{b}, \tilde{c})$ is the free lattice generated by its subposet $\{a, b, c\}$.

We are going to use the two lemmas above in the proof of the following lemma. Implicitly, we will often use the well-known Homomorphism Theorem; see, e.g., Burris and Sankappanavar [1, Theorem 6.12].

Lemma 5. If an n-element lattice $L$ has a 3-antichain, then we have that $|\operatorname{Sub}(L)| \leq 20 \cdot 2^{n-5}$.

Proof. Let $\{a, b, c\}$ be a 3 -antichain in $L$. Lemma 3 yields a unique join-homomorphism from $F_{\mathrm{jl}}((\tilde{a}, \tilde{b}, \tilde{c}))$ to $S:=\{a, b, c, a \vee b, a \vee c, b \vee c, a \vee b \vee c\}$ such that $\varphi$ maps to $\tilde{a}, \tilde{b}$, and $\tilde{c}$ to $a, b$, and $c$, respectively. Since $\{a, b, c\}$ is an antichain, none of the six lower edges of $F_{\text {jl }}((\tilde{a}, \tilde{b}, \tilde{c}))$ is collapsed by the $\operatorname{kernel} \Theta:=\operatorname{ker}(\varphi)$ of $\varphi$. Hence, there are only four cases for the join-subsemilattice $S \cong F_{\mathrm{jl}}((\tilde{a}, \tilde{b}, \tilde{c})) / \Theta$ of $L$, depending on the number the upper edges collapsed by $\Theta$.

Case 1: [none of the three upper edges is collapsed by $\Theta$ ] Then $S$ is isomorphic to $F_{\text {js }}((\tilde{a}, \tilde{b}, \tilde{c}))$, whereby $\{a \vee b, a \vee c, b \vee c\}$ is a 3-antichain. We know from, say, Grätzer [5, Lemma 73], that this 3-antichain generates a sublattice isomorphic to $B_{8}$. Hence, $|\operatorname{Sub}(L)| \leq 9.25 \cdot 2^{n-5} \leq 20 \cdot 2^{n-5}$ by Lemmas $1(2)$ and 2(7), as required.
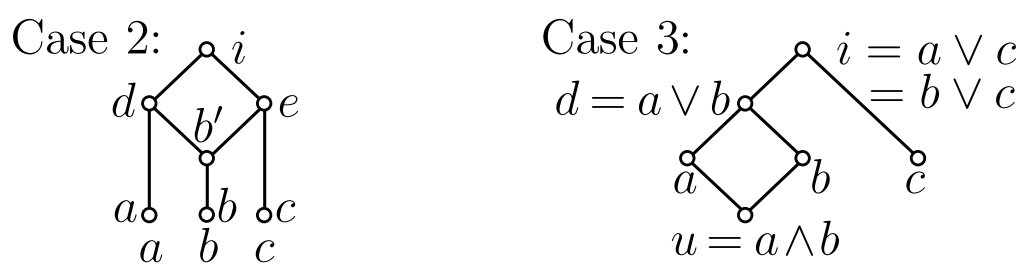

FiguRE 4. Cases 2 and 3

Case 2: $[\Theta$ collapses exactly one upper edge] Apart from notation, we have that $d:=a \vee b<a \vee c=: i$ and $e:=b \vee c<i$; see Figure 4 on the left. Letting $b^{\prime}:=d \wedge e$, we have that $a \vee b^{\prime}=d$ and $b^{\prime} \vee c=e$. Since $b \leq b^{\prime}$ and $b \not \leq a$, we have that $b^{\prime} \not \leq a$. If we had $a \leq b^{\prime}$, then $i=d \vee e=a \vee b^{\prime} \vee e=b^{\prime} \vee e=e$ would be a contradiction. Hence, $a \| b^{\prime}$, and $\left\{a, b^{\prime}, c\right\}$ is a 3 -antichain by $a-c$ symmetry. We can count the subuniverses $T$ of the join-semilattice $H:=$ $\left\{a, b^{\prime}, c, d, e, i\right\}$ as follows. We have that $\left|\left\{T: b^{\prime} \notin T\right\}\right| \leq 3 \cdot 7=21$, because $\{d, e\} \nsubseteq T$ allows only three possibilities for $T \cap\{d, e\}$ and $a \vee c=i$ at most seven possibilities for $T \cap\{a, c, i\}$. Similarly,

$$
\begin{aligned}
& \left|\left\{T: b^{\prime} \in T, a \notin T, c \notin T\right\}\right| \leq 7, \quad \text { because }\{d, e\} \subseteq T \Rightarrow i \in T, \\
& \left|\left\{T: b^{\prime} \in T, a \in T, c \notin T\right\}\right| \leq 3, \quad \text { since } d \in T, \text { so } e \in T \Rightarrow i \in T,
\end{aligned}
$$


$\left|\left\{T: b^{\prime} \in T, a \notin T, c \in T\right\}\right| \leq 3, \quad$ by $a-c$ symmetry, and $\left|\left\{T: b^{\prime} \in T, a \in T, c \in T\right\}\right|=1$, because $T=H$.

Note that some of the inequalities above are equalities, but we do not need this fact. Forming the sum of the above numbers, the join-semilattice $H$ has at most $35=17.5 \cdot 2^{6-5}$ subuniverses. Hence, Lemma 1(1) yields that $\operatorname{Sub}(L) \leq 17.5 \cdot 2^{n-5} \leq 20 \cdot 2^{n-5}$, as required.

Case 3: $[\Theta$ collapses two of the upper edges] Apart from notation, we have that $d:=a \vee b<a \vee c=b \vee c=: i$. Let $u:=a \wedge b$; see Figure 4. We focus on possible intersections of subuniverses of $L$ with $H:=\{a, b, c, d, u, i\}$. Denoting such an intersection by $S$, we can compute as follows.

$$
\begin{aligned}
& |\{S: c \notin S\}| \leq 26, \quad \text { by Lemmas } 1(2) \text { and } 2(1), \\
& |\{S: c \in S, a \in S, b \in S\}| \leq 1 \quad \text { since } H \subseteq[\{a, b, c\}], \\
& |\{S: c \in S, a \in S, b \notin S\}| \leq 4 \quad \text { since } i \in[\{a, c\}], \\
& |\{S: c \in S, a \notin S, b \in S\}| \leq 4, \quad \text { by } a-b \text {-symmetry, } \\
& |\{S: c \in S, a \notin S, b \notin S, d \in S\}| \leq 2, \quad \text { because } i \in S, \\
& |\{S: c \in S, a \notin S, b \notin S, d \notin S\}| \leq 3 \quad \text { since } u \in S \Rightarrow i \in S .
\end{aligned}
$$

Since the sum of these numbers is 40 , we obtain from Lemma 1(1) that $|\operatorname{Sub}(L)| \leq 40 \cdot 2^{n-6}=20 \cdot 2^{n-5}$, as required.

Case 4: [all the three upper edges are collapsed] Clearly, $a \vee b=a \vee c=b \vee$ $c=a \vee b \vee c=: i$. If $a \wedge b=a \wedge c=b \wedge c=a \wedge b \wedge c$ failed, then the dual of one of the previous three cases would apply. Hence, we can assume that the sublattice $[\{a, b, c\}]$ generated by $\{a, b, c\}$ is isomorphic to $M_{3}$; see Figure 2 . Therefore, $|\operatorname{Sub}(L)| \leq 20 \cdot 2^{n-5}$ by Lemmas $1(2)$ and 2(6), completing the proof of Case 4 and that of Lemma 5.

Proof of Theorem 1. From Lemmas 1 and 2(1), we conclude part (1). So, we are left only with part (2).

In what follows, let $L$ be an $n$-element lattice. We obtain from Lemmas 1(3) and 2(2) that if

$$
L \cong C_{0}+{ }_{g l u} N_{5}+{ }_{g l u} C_{1} \text { for finite chains } C_{0} \text { and } C_{1},
$$

then $|\operatorname{Sub}(L)|=23 \cdot 2^{n-5}$. In order the complete the proof of Theorem 1, it suffices to exclude the existence of a lattice $L$ such that

$$
\begin{aligned}
& |L|=n, 23 \cdot 2^{n-5} \leq|\operatorname{Sub}(L)|<26 \cdot 2^{n-5} \text {, but } L \text { is } \\
& \text { not of the form given in (3.1). }
\end{aligned}
$$

Suppose, for a contradiction, that $L$ is a lattice satisfying (3.2). Then, by (1.1) and Theorem 1 (1) and Lemma 5,

$L$ has at least two 2-antichains but it has no 3-antichain. 
We claim that

Suppose the contrary. Then we can pick two distinct antichains $\{a, b\}$ and $\{c, b\}$ in $L$. Since there is no 3 -antichain in $L$, we can assume that $a<c$. With $K:=[\{a, b, c\}]$, let $\varphi: F_{\text {lat }}(\tilde{a}, \tilde{b}, \tilde{c}) \rightarrow K$ be the unique lattice homomorphism from Lemma 4 , and let $\Theta$ be the kernel of $\varphi$. We claim that $\Theta$ collapses $e_{1}$; see Figure 3. Suppose to the contrary that $\Theta$ does not collapse $e_{1}$. Since $e_{1}$ generates the monolith congruence, that is, the smallest nontrivial congruence of the $N_{5}$ sublattice of $F_{\text {lat }}(\tilde{a}, \tilde{b}, \tilde{c})$, no other edge of this $N_{5}$ sublattice is collapsed. Hence, $N_{5}$ is a sublattice of $L$, and it follows from Lemmas $1(2)$ and 2(2) that $|\operatorname{Sub}(L)| \leq 23 \cdot 2^{n-5}$. Thus, (3.2) yields that $|\operatorname{Sub}(L)|=23 \cdot 2^{n-5}$. Applying Lemma $1(3)$ for $K:=N_{5}$ and $L$, we obtain that $L$ is of the form (3.1). This contradicts (3.2), and we have shown that $\Theta$ does collapse $e_{1}$. On the other hand, since $a \| b$ and $c \| b$, none of the thick edges $e_{8}, \ldots, e_{11}$ is collapsed by $\Theta$. Observe that at least one of $e_{4}$ and $e_{6}$ is not collapsed by $\Theta$, since otherwise $\langle\tilde{a}, \tilde{c}\rangle$ would belong to $\Theta=\operatorname{ker}(\varphi)$ by transitivity and $a=c$ would be a contradiction. By duality, we can assume that $e_{4}$ is not collapsed by $\Theta$. Since $e_{2}, e_{3}$, and $e_{5}$ are perspective to $e_{10}, e_{9}$, and $e_{4}$, respectively, these edges are not collapsed either. So, with the exception of $e_{1}$, no edge among the "big" elements in Figure 3 is collapsed. Thus, the $\varphi$-images of the elements denoted by big circles form a sublattice (isomorphic to) $C^{(2)} \times C^{(3)}$ in $L$. Hence, $|\operatorname{Sub}(L)| \leq 19 \cdot 2^{n-5}$ by Lemmas 1(2) and 2(3), which contradicts our assumption that $L$ satisfies (3.2). This proves (3.4).

To provide a convenient tool to exploit (3.3) and (3.4), we claim that

$$
\begin{aligned}
& \text { if } x, y, z \in L \text { such that }|\{x, y, z\}|=3 \text { and } x \| y \text {, then either } \\
& \{x, y\} \subseteq \downarrow z \text {, or }\{x, y\} \subseteq \uparrow z .
\end{aligned}
$$

To see this, assume the premise. Since $L$ has no 3-antichain, $z$ is comparable to one of $x$ and $y$. By duality and symmetry, we can assume that $x<z$. Since $z<y$ would imply $x<y$ and $z \| y$ together with $x \| y$ would contradict (3.4), we have that $y<z$. This proves (3.5).

Next, by (3.3) and (3.4), we have a four-element subset $\{a, b, c, d\}$ of $L$ such that $a \| b$ and $c \| d$. By duality and (3.5), we can assume that $\{a, b\} \subseteq \downarrow c$. Applying (3.5) also to $\{a, b, d\}$, we obtain that $\{a, b\}$ is included either in $\uparrow d$, or in $\downarrow d$. Since the first alternative would lead to $d<a<c$ and so it would contradict $c \| d$, we have that $\{a, b\} \subseteq \downarrow d$. Thus, $\{a, b\} \subseteq \downarrow c \cap \downarrow d=\downarrow(c \wedge d)$, and we obtain that $u:=a \vee b \leq$ $c \wedge d=: v$. Let $S:=\{a \wedge b, a, b, u, v, c, d, c \vee d\}$. Depending on $u=v$ or $u<v, \bar{S}$ is a sublattice isomorphic to $B_{4}+{ }_{g l u} B_{4}$ or $B_{4}+{ }_{g l u} C^{(2)}+{ }_{g l u} B_{4}$. Using Lemma 2.1 together with (4) and (5) of Lemma 2, we obtain that $|\operatorname{Sub}(L)| \leq 21.25 \cdot 2^{n-5}$. This inequality contradicts (3.2) and completes the proof of Theorem 1. 


\section{REFERENCES}

[1] S. Burris and H. P. Sankappanavar, A Course in Universal Algebra. New York-Berlin: Springer, 1981. [Online]. Available: http://www.math.uwaterloo.ca/ \{\} snburris/htdocs/ualg.html. doi: 10.1080/00029890.1984.11971342

[2] G. Czédli, "A note on finite lattices with many congruences.” Acta Univ. M. Belii Ser. Math., pp. 22-28, 2018. [Online]. Available: http://actamath.savbb.sk/pdf/oacta2018003.pdf

[3] G. Czédli, "Lattices with many congruences are planar." Algebra Universalis, p. 80:16, 2019, doi: 10.1007/s00012-019-0589-1.

[4] R. Freese, "Computing congruence lattices of finite lattices." Proc. Amer. Math. Soc., vol. 125, pp. 3457-3463, 1997, doi: 10.1090/S0002-9939-97-04332-3.

[5] G. Grätzer, Lattice Theory: Foundation. New York: Birkhäuser Verlag, 2011. [Online]. Available: tinyurl.com/lattices101. doi: 10.1007/978-3-0348-0018-1

[6] J. Kulin and C. Mureşan, "Some extremal values of the number of congruences of a finite lattice." 2018. [Online]. Available: https://arxiv.org/pdf/1801.05282

[7] I. Rival and R. Wille, "Lattices freely generated by partially ordered sets: which can be "drawn"?" J. Reine Angew. Math., vol. 310, pp. 56-80, 1979, doi: 10.1515/crll.1979.310.56.

Authors' addresses

Gábor Czédli

University of Szeged, Bolyai Institute, Aradi vértanúk tere 1, 6720 Szeged, Hungary

E-mail address: czedli@math.u-szeged.hu

Eszter K. Horváth

University of Szeged, Bolyai Institute, Aradi vértanúk tere 1, 6720 Szeged, Hungary

E-mail address: horeszt@math.u-szeged.hu 